\title{
The indirect self-tuning method for constructing radio environment map using omnidirectional or directional transmitter antenna
}

\author{
Marko Pesko ${ }^{1,4^{*} \dagger}$, Tomaž Javornik $2,4 \dagger$, Luka Vidmar ${ }^{1}$, Andrej Košir ${ }^{3}$, Mitja Štular ${ }^{1}$ and Mihael Mohorčič $2,4 \dagger$
}

\begin{abstract}
In this paper, we propose a new indirect method for constructing the radio frequency layer of radio environment map (RF-REM) called self-tuning method (STM). The proposed STM takes into account the characteristics of the operating environment and performs estimation of the transmitter parameters, i.e. its location, antenna diagram, antenna azimuth, transmit power, as well the parameters of the propagation model to obtain the best match between the available measurements and the predicted signal levels. We compare STM to several most often considered existing methods using different numbers of randomly distributed measurements, which could in practice be obtained in a participatory-like manner. The performance evaluation of the methods is performed in terms of averaged root mean square error ( $\overline{\mathrm{RMSE}}$ ) and 95\% confidence interval (CI) calculated between the constructed RF-REMs and the reference RF-REM, as well as in terms of false alarm zone ratio (FAZR) and correct detection zone ratio $\left(C D Z R_{1}\right)$. The analysis shows the robustness of the STM to various spatial distributions of measurements and its fast convergence and low residual root mean square error compared to the inverse distance weighted (IDW) method, the inverse distance square weighted method (IDW2), the Kriging method, and the location estimation-based method (LIVE).
\end{abstract}

Keywords: Cognitive radio; REM; RF-REM; Construction methods; Mobile network

\section{Introduction}

The nature of radio signal propagation imposes on wireless communication systems to share the radio frequency (RF) spectrum in time and space. Traditionally, the publicly accessible spectrum is divided into the licensed and the unlicensed frequency bands. This arrangement is becoming inefficient because more and more radio resources are needed in geographically limited areas to meet the increasing capacity demand, calling for improved spectrum utilization. In fact, several measurement campaigns revealed that many parts of the licensed frequency bands are often underutilized, while the unlicensed bands are saturated in time and space [1]. This

\footnotetext{
${ }^{*}$ Correspondence: marko.pesko@telekom.si

†Equal contributors

1 Telekom Slovenije, d.d., Cigaletova 15, 1000 Ljubljana, Slovenia

${ }^{4}$ Jozef Stefan International Postgraduate School, Jamova cesta 39, 1000

Ljubljana, Slovenia

Full list of author information is available at the end of the article
}

observation led to the emergence of dynamic spectrum access (DSA) approach, which enables (through the concept of cognitive radio (CR)) more efficient spectrum usage [2], by exploiting the flexibility of software-defined radio (SDR). The basic precondition for the device that makes use of the DSA approach is spectrum awareness, which can be achieved either by the built-in spectrum sensing capability or by looking-up in a purposely deployed and maintained geolocation database of available radio resources [1].

Research and development of the spectrum sensing techniques have revealed that the low-cost spectrum sensing devices cannot yet fulfil the sensitivity criteria for a reliable detection of radio signals at signal levels required by Federal Communications Commission (FCC) [3] and Electronic Communications Committee (ECC) [4]. One proposed solution to overcome the need for high precision spectrum sensing on each device is a radio environment map (REM) comprising multi-domain information

\section{是 Springer}

(c) 2015 Pesko et al: licensee Springer. This is an Open Access article distributed under the terms of the Creative Commons

Attribution License (http://creativecommons.org/licenses/by/4.0), which permits unrestricted use, distribution, and reproduction in any medium, provided the original work is properly credited. 
from geolocation databases [1] to allow sharing of unused spectrum primarily allocated to the television broadcast services [5]. It is also considered to support hierarchical spectrum access on licensed bands, spectrum sharing on unlicensed bands, intra-operator radio resource management, and dedicated spectrum monitoring [6]. To support such scenarios, REM must provide comprehensive up to date representation of the radio environment with its selectable and adaptable content details. This is used to depict past, current, as well as expected conditions in the area of interest and can be classified into three main categories or layers related to (i) radio elements, (ii) radio scene, and (iii) radio environment [7]. Furthermore, REM content can be divided into long-term information and short-term information. The first one is slowly changing, it may be also quasi-static or static. It is related to TV broadcast and receiver $(\mathrm{Rx})$ stations, mobile operator base stations (BSs), and other similar transmitters (Tx). On the contrary, the period of updating short-term information, such as information about wireless microphones on TV frequencies and other mobile Txs, can be much shorter. The most important REM content is the interference information [7] which can be estimated from maps representing the coverage of an area with the radio signal, named RF-REM layers or RF-REMs. These are sub-layers of the radio environment layer and in the most basic form present the coverage of an area with the radio signal at a specific frequency. In the literature, RF-REMs are also referred to as RF environment maps (RFEMs), radio electric exposure level maps (REELMs), power spectral density maps (PSDs), and most often simplified as REMs, although the latter term is broadly used for the entire REM concept. Today, the signal measurements required for RF-REM construction are obtained by dedicated static sensors or by periodic measurement campaigns, which results in a static or quasi-static RFREM. However, future radio environment when DSA is supported will be dynamic with heterogeneous wireless systems competing for the available shared spectrum. In such radio environment, the existing approaches for spectrum measurement will have to be complemented by distributed spectrum sensing. To this end, a particularly well-suited approach to the operators of wireless systems is spectrum sensing by advanced user terminals with spectrum sensing capability operating in participatory manner or dense but irregular deployments of low-cost less sensitive spectrum sensors. With such an approach, signal level measurements will be provided by heterogeneous measurement capable devices. The existing RF-REM construction methods are not designed for signal measurements obtained in participatory manner, where data is provided dynamically in irregular time intervals from random locations. They do not explicitly take into account the operating environment characteristics, and they rely on large sets of signal measurements to achieve suitable accuracy.

The main contribution of this paper is a new indirect RF-REM construction method that is well-suited also to collection of measurements points using a participatory sensing approach. Its characteristics can be summarized as follows: (i) the method takes into account the characteristics of the Tx, i.e. transmit power, antenna diagram, its azimuth and tilt, (ii) the signal measurements are applied for the propagation model calibration, and (iii) the method relies on the semi-empirical propagation model in which the empirical propagation model is complemented with the available information about the radio propagation environment including the digital elevation model and clutter information of the geographical area of interest. We perform a systematic investigation of the impact of the number and distribution of measurements in realistic directional Tx scenario and evaluate the proposed RF-REM construction method in comparison to several existing RF-REM construction methods by using averaged root mean square error ( $\overline{\mathrm{RMSE}})$ as the performance metric. Applying the RMSE metric shows that the proposed method outperforms most often cited methods for different spatial distributions of signal measurements. Furthermore, we show that the existing typically used performance metrics can yield wrong interpretation of the results when dynamic scenario is considered, since they do not take into account the different spatial distributions of measurements available for RF-REM construction in a given time moment. Nevertheless, we evaluated the considered RF-REM construction method also in terms of correct detection zone ratio $\left(\mathrm{CDZR}_{1}\right)$ and false alarm zone ratio (FAZR) for the representative number of measurements.

The rest of this paper is organized as follows. We introduce the RF-REM construction in Section 2 and describe the proposed STM in Section 3. The implementation setup and simulation scenario definition are provided in Section 4, whereas performance evaluation of the considered construction methods is presented and discussed in Section 5. Finally, Section 6 concludes the paper.

\section{RF-REM construction}

The construction of REM can be in a most basic form presented as a construction of RF-REM and deals with estimating the signal levels at distributed geographical locations where signal measurements are not available. The RF-REM construction methods can be classified into (i) direct methods applying interpolation, (ii) indirect methods utilizing Tx parameters and propagation modelling [8], and (iii) hybrid methods which combine both approaches.

The direct construction methods are based on local neighbourhood, geostatistical, and variational interpo- 
lation [9]. The most widely RF-REM construction applied interpolation methods for RF-REM construction found in the literature are the inverse distance weighted (IDW) method [10], the nearest neighbours (NN) method [11], the splines methods [12], modification and derivation of mentioned methods such as the modified Shepard's method (MSM) [13], the gradient plus inverse distance squared (GIDS) method [7], and the Kriging method [14]. Among these, the Kriging method is most often applied for the direct RF-REM construction, since it is the best geostatistical linear unbiased estimator that yields a zero mean residual error and minimizes the error variance.

In the indirect RF-REM construction methods, a Tx location and/or its parameters are estimated first, and then, the RF-REM is calculated using radio propagation modelling. These methods are often computationally more complex and not as commonly used as the direct methods. Nevertheless, they can be used to describe the RF coverage more realistically, especially in the long-term information REM where RF-REM construction time is not of such great importance as in the short-term information REM. The indirect methods distinguish three scenarios where (i) location and other parameters of the Tx are known; (ii) either the Tx location or its parameters are known; (iii) both, Tx location and its parameters, are unknown. In (i), the RF-REM construction is straightforward and it is reduced to the calculation of the RF signal coverage with a propagation model using known Tx parameters. This approach is also used for the planning of wireless networks. In (ii), an efficient estimation of the Tx location is performed if other parameters of Tx such as the transmit power, the antenna characteristics etc. are known. After estimating the Tx location, the approach outlined for the first case can be applied. On the other hand, if only the location is known, the Tx power, antenna characteristics and other Tx parameters are estimated by modelling the antenna diagram and suitable propagation model calibration. In (iii), localization of Tx, estimation of its parameters, and calibration of the propagation model, if considered, are performed before performing the RF-REM construction with propagation modelling. The recent research in indirect methods $[1,15]$ follows this idea by proposing the Tx location estimation-based REM construction method (LIvE). By providing more accurate RF-REM than the direct Kriging method, LIvE indicates further research possibilities in the direction of indirect construction methods.

The hybrid RF-REM construction combines direct and indirect approaches. For example, in [11], various interpolation methods are used first, to interpolate measurements into an image, which is in the case of direct methods already the RF-REM. Then, identification of propagation and Tx features is performed by using image processing techniques. Finally, propagation modelling is used to construct final RF-REM. A different method is proposed in [16], where at first, a preliminary RF-REM is constructed by a simple numerical propagation modelling, which is in the next step corrected according to the available measurements with the direct construction approach.

The results from [1] suggest that the LIvE method, which relies on an empirical propagation model obtained from the statistical analysis of a large number of measurements and highly dependent on the adaptation to a specific geographical area of interest, outperforms most often considered direct methods IDW, IDW2, and Kriging. However, none of these methods is well suited for consideration of real operating scenarios as they do not explicitly take into account a priori knowledge available about the characteristics of the operating environment, nor they consider the Tx directivity. Namely, in real operating scenarios with directional Txs or with omnidirectional Txs whose radiation is heavily obstructed or completely blocked in specific azimuthal direction, appropriate modelling of Tx directivity is very important.

Both concerns can be taken into account by using deterministic and semi-empirical propagation models [17]. If properly selected with respect to the operating environment, they are usually more accurate than the empirical models and thus more often considered in practice. The deterministic models require an enormous number of operating environment information and huge computational effort, while semi-empirical models obtained as their empirical modifications have a moderate complexity.

Our hypothesis is that an indirect RF-REM construction method based on a semi-empirical propagation model, taking into account a priori knowledge about characteristics of the operating environment and modelling the antenna influence of the Tx, can enhance the accuracy of current RF-REM construction assuming (i) slow changing long-term information REM scenario, and (ii) that the knowledge about the operating environment is available for RF-REM construction. In the next section, we propose a new indirect self-tuning method (STM) which is following such an approach. It should be noted that STM itself is not a specific algorithm for RF-REM construction, since each step of the STM can be implemented and solved in various ways.

\section{STM}

The STM consists of three main steps: (i) the data collection; (ii) the Tx parameters' estimation and propagation model calibration; and (iii) the RF-REM construction.

\section{Step 1}

In the first step, signal measurements for the RF-REM construction as well as supplementary data, such as a digital model of the terrain and the corresponding 
clutter loss data of the geographical area of interest, are collected.

\section{Step 2}

The second step concerns approximation of the Tx location, calibration of the propagation model, and an estimation of Tx parameters. This can be in practice performed with various algorithms on different hardware platforms, so in the following, we only provide one possible implementation.

Let the pair $(x, y)$ reflects location coordinates. Let us further assume that in the area of interest for constructing RF-REM, there are $N$ measurements at points (pixels) which can be denoted as $R=\left\{R_{i}=\left(x_{i}, y_{i}\right), i\right\}$. Similarly, Tx location or an area of possible Tx locations can be represented as $T=\left\{T_{j}=\left(x_{j}, y_{j}\right), j\right\}$. T can be pre-known or calculated using a suitable localization algorithm (e.g. based on individual or combined localization technique such as angle of arrival (AA), timing advance (TA), time of arrival (TOA), time difference of arrival (TDOA) etc.). The output of such algorithm is usually an estimated location or an area of possible Tx locations, where the Tx can be found with a given probability. The received power $P_{\mathrm{rx}_{i}}$ $[\mathrm{dBm}]$ at $i$-th Rx location can be estimated as in (1), where $P_{t x}[\mathrm{dBm}]$ is the transmit power and $L_{\mathrm{rx}_{i}}[\mathrm{~dB}]$ is the corresponding path loss for the operating environment and frequency band.

$$
P_{\mathrm{rx}_{i}}=P_{\mathrm{tx}}-L_{\mathrm{rx}_{i}}=P_{\mathrm{tx}}-\left(L_{i}+L_{\mathrm{diff}_{\mathrm{i}}}+L_{\mathrm{clut}_{i}}-G_{\mathrm{ant}_{i}}\right) .
$$

In this paper, the sub-GHz mobile communication systems band is considered as a reference, so we take the semi-empirical modification of the Okumura-Hata model implemented as in [18]. Thus, $L_{\mathrm{rx} i}$ is a sum of the path loss of the empirical channel model $L_{i}$, diffraction loss $L_{\text {diff }}$, loss due to terrain clutter $L_{\mathrm{clut}_{i}}$, and loss due to the directional gain $G_{\mathrm{ant}_{i}}$ of the Tx antenna. $L_{i}$ follows modified Okumura-Hata model for open areas (2), where $d[\mathrm{~m}]$ is the distance between $T_{j}$ and $R_{i}, H_{\text {eff }_{i}}[\mathrm{~m}]$ is the Tx effective antenna height [18] dependent on the Tx and Rx antenna height, $f[\mathrm{MHz}]$ is the carrier frequency, $A_{0}$ is the offset, and $A_{1}, A_{2}$, and $A_{3}$ are slope coefficients of the path loss.

$$
\begin{aligned}
L_{i}= & A_{0}+A_{1} \log _{10}(d)+A_{2} \log _{10}\left(H_{\mathrm{eff}_{i}}\right) \\
& +A_{3} \log _{10}(d) \log _{10}\left(H_{\mathrm{eff}_{i}}\right) \\
& -3.2\left[\log _{10}\left(11.75 H_{m}\right)\right]^{2} \\
& +44.49 \log _{10}(f)-4.78\left[\log _{10}(f)\right]^{2} .
\end{aligned}
$$

$L_{\text {diff }_{i}}$ is the loss calculated as a knife edge effect on the main obstacle obstructing the radius of the first Fresnel zone, based on the elevations of the terrain. $L_{\mathrm{clut}_{i}}$ is queried directly from the REM storage or an available external database. $G_{\text {ant }_{i}}$ represents generalized antenna pattern, where $\theta_{i}$ is the horizontal and $\phi_{i}$ is the vertical angle between Tx at $T_{j}$ and $i$-th $\mathrm{Rx}$ at $R_{i}$. It assumes symmetrical or at least approximately symmetrical horizontal and vertical antenna pattern of the Tx to the bore sight direction $\theta_{0}$ and $\phi_{0}$. In Equation 3, $\theta_{0}$ and $\phi_{0}$ are the antenna boresight directions, $G_{\max }$ is the maximum antenna gain, and $F B R_{\theta}$ as well as $F B R_{\phi}$ are front to back ratios, whereas the antenna form is approximated with the cosine functions to the power of $m$ and $n$.

$$
\begin{aligned}
G_{\mathrm{ant}_{i}}=G_{\mathrm{max}} & -F B R_{\theta}+F B R_{\theta}\left|\cos ^{m}\left(\frac{\theta_{0}-\theta_{i}}{2}\right)\right| \\
- & F B R_{\phi}+F B R_{\phi}\left|\cos ^{n}\left(\frac{\phi_{0}-\phi_{i}}{2}\right)\right| .
\end{aligned}
$$

Now we define a vector $\beta$ (4) consisting of parameters of the empirical propagation model (2) and parameters from Equation 3.

$$
\vec{\beta}=\left[A_{0}, A_{1}, A_{2}, A_{3}, G_{\max }, F B R_{\theta}, \theta_{0}, m, F B R_{\phi}, \phi_{0}, n\right]^{T} .
$$

As given in (5), the STM finds such $j$ (i.e. $T_{j}$ from $T$ ) and $\vec{\beta}$ that minimize the mean squared error between the measured and estimated signal strengths. In (5), $N$ is the number of Rx locations, $P_{i}$ denotes measured signal level and $L_{\mathrm{rx}_{i}}$ the semi-empirically estimated path loss at the location of the $i$-th $\mathrm{Rx}$, considering the Tx with $P_{\mathrm{tx}}$ at $T_{j}$.

$$
F(\vec{\beta}, j)=\frac{1}{N} \sum_{i=1}^{N}\left(P_{i}-\left(P_{\mathrm{tx}}-L_{\mathrm{rx}_{i}}\left(\vec{\beta}\left(T_{j}\right)\right)\right)\right)^{2} .
$$

The non-linear optimization problem with known typical lower and upper bounds can be solved in terms of $\left(\vec{\beta}^{*}, j^{*}\right)=\min _{\vec{\beta}, j} F(\vec{\beta}, j)$ by a suitable optimization method for solving non-linear optimization problems. However, if the directivity of the Tx antenna given in (3) is neglected, i.e. if the omnidirectional Tx antenna is considered, then $G_{\text {ant }}$ is constant and the optimization objective (5) becomes linear and can be solved by a suitable optimization method for linear optimization problems.

\section{Step 3}

After solving the optimization problem, the estimated values for $\vec{\beta}^{*}, T_{j}^{*}$, and $P_{\mathrm{tx}}$ are applied in the calculation of a signal level on individual RF-REM location according to $(6)$.

$$
P_{\mathrm{rx}_{i}}=\widehat{P}_{\mathrm{tx}}-\widehat{L}_{\mathrm{rx}_{i}}=\widehat{P}_{\mathrm{tx}}-\left(\widehat{L}_{i}+L_{\mathrm{diff}_{i}}+L_{\mathrm{clut}_{i}}-\widehat{G}_{\mathrm{ant}_{i}}\right) .
$$


4 Implementation setup and scenario definition

In this section, we describe the reference implementation setup and operating scenario of the proposed STM as considered in subsequent performance evaluation.

\subsection{Reference RF-REM}

For the performance evaluation of different RF-REM construction methods, we need a reference RF-REM, i.e. a reference signal coverage distribution. To this end, we consider a representative real rectangular geographical area within Slovenia depicted in Figure 1. The size of the area is $5.15 \times 6.75 \mathrm{~km}$ and is represented with digital elevation model with the resolution of $25 \mathrm{~m}$. It consists of nine different clutter categories. In the area, we consider a single active Tx for a single channel, similar as in [1]. It is positioned at the location of a BS operating in the 900-MHz frequency band. We take into account the actual BS settings provided by the mobile operator: the transmit power of $39 \mathrm{dBm}$, Kathrein K739650 antenna pattern with $35^{\circ}$ of azimuth, and mechanical down-tilt of $2^{\circ}$. The initial approximation of signal levels is depicted in Figure 2, where the reference RF-REM has been calculated down to the threshold of $-120 \mathrm{dBm}$ with the wireless network planning tool GRASS RaPlaT [19], which was calibrated to the drive-test measurements of the mobile operator taking into account the clutter as well as the shadowing based on the terrain elevation data. Thus, it can be assumed that the calculated signal level at each $25 \times 25 \mathrm{~m} \mathrm{RF-REM} \mathrm{point} \mathrm{is}$ very close to real situation.

\subsection{Scenarios and measurements spatial distributions}

In order to model distributed measurement sets of various sizes and spatial distributions, as if obtained in a participatory manner, the reference RF-REM was sampled at random locations. Since GRASS RaPlaT cannot introduce random errors at simulated RF-REM points, this has been subsequently added to the sampled signal levels at RF-REM points as random values obeying a log-normal distribution with mean $0 \mathrm{~dB}$ and standard deviation of $8 \mathrm{~dB}$. The probability density function, its standard deviation and mean value, has been obtained by analysing measurements collected on the field by the mobile operator. We found this sufficiently approximates the real distribution of measurements at individual RFREM points. Finally, such participatory measurement sets were used for the evaluation of the considered RF-REM construction methods.

When measurements for RF-REM construction are obtained in a participatory manner, the number and spatial distribution of available measurements in a set may vary. Consequently, for fair comparison of the performance of different RF-REM construction methods, the methods should be tested for different scenarios which can occur in practice.

Since measurements can be randomly distributed in the entire area or only in its sub-regions, we consider three simulation scenarios for modelling different situations and thus investigate the impact of spatial distribution of measurements with respect to Tx antenna main lobe on the accuracy of the RF-REM construction methods. In order to test the impact of the number and distribution of participatory measurements on the RF-REM construction methods, we sampled the reference RF-REM and generated 100 different measurement sets of the specific size by increasing the number of measurement points (and hence their density) from 10 to 1,000. In the 'reference' scenario I, measurements are randomly distributed in the entire area of interest, as depicted in Figure 3a. In the scenario II, the majority (90\%) of the measurements are inside the main lobe of the antenna, as is illustrated in Figure 3b. The purpose of this scenario is to test the need for anchor measurement points in the RF-REM construction of a

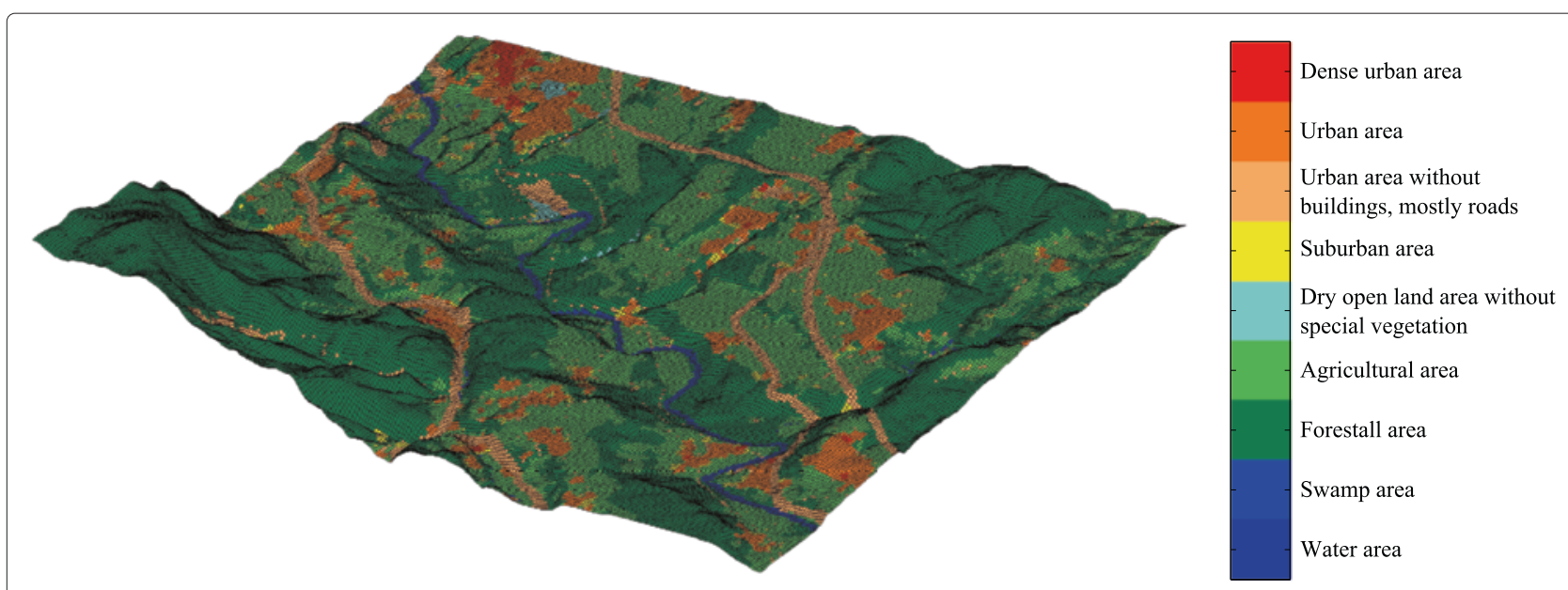

Figure 1 The geographical area with nine different clutter categories. 


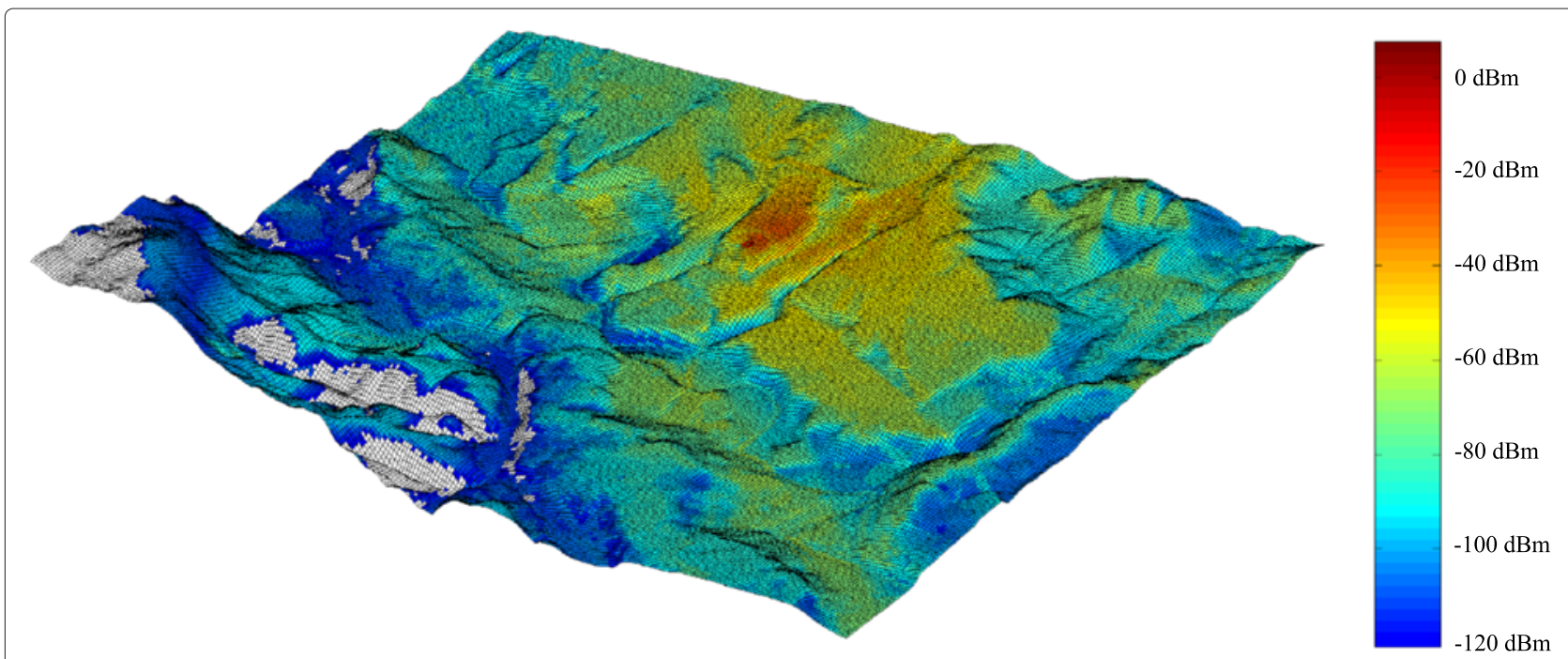

Figure 2 The initial approximation of signal levels in the area.

directional Tx. In practice, however, the location of measurements depends on the operating environment, and thus, they can be found in certain clutter category more likely than in the others. In this respect, for the scenario III shown in Figure 3c, measurements are distributed according to clutter categories. The aim of this scenario is not to simulate real distribution of measurements over clutter but rather to investigate the impact of measurements' clustering. To this end, we define the distribution of measurements regarding the clutter categories and assume that measurements in water, i.e. rivers and lakes, and swamp areas are not available or their number can be neglected. For other clutter categories, we assume most measurements in dense urban areas (30\%), followed by urban areas $(25 \%)$, urban areas without buildings, i.e. mostly roads, (20\%), sub-urban areas (10\%), dry open land areas without special vegetation (7\%), agricultural areas (5\%), and forestall areas (3\%).

\subsection{Implementation of the methods}

We implemented the IDW, IDW2, Kriging, LIvE, and STM methods in Matlab and tested them using the same measurement sets. For the STM analysis, we considered two cases, namely having a transmitter equipped with omnidirectional antenna or with directional antenna. Considering the omnidirectional antenna, we solved the linear optimization problem without optimization bounds in the well-defined least square sense by using the Matlab function mldivide(), similar as the LIvE method for the omnidirectional Tx case.

For the second STM case study, which includes the directivity of the Tx antenna, we considered only the horizontal part of the antenna pattern in the optimization process. This simplification significantly reduces the computational complexity and improves the convergence of optimization process; at the same time, it does not introduce significant deviation, assuming the measurements

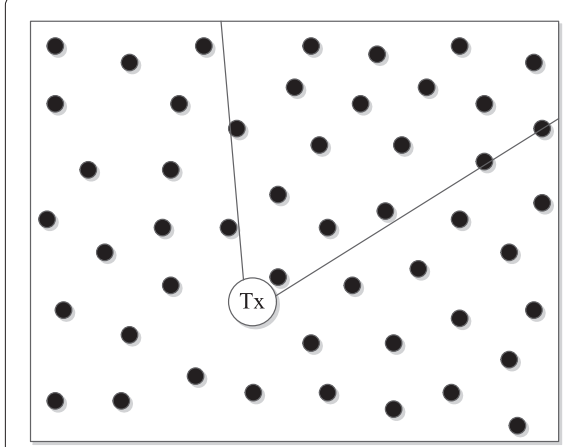

(a)

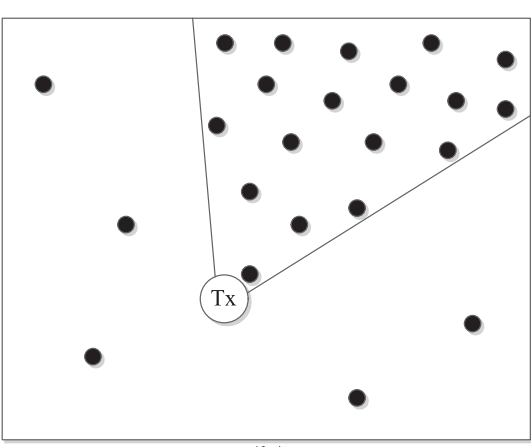

(b)

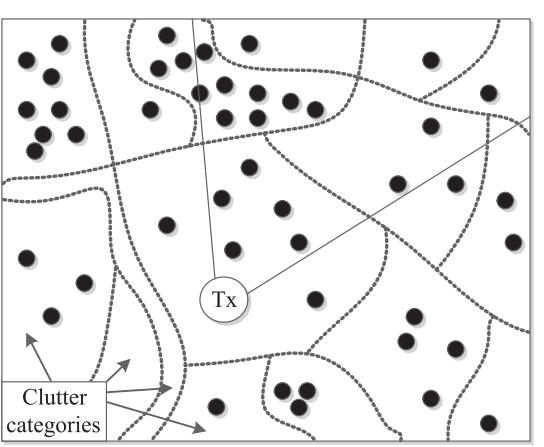

(c)

Figure 3 Distribution of measurements in considered scenarios. (a) Scenario I - randomly in space, (b) scenario II - $90 \%$ in the main lobe of the antenna, and (c) scenario III - clutter-dependent clustered distribution. 
are within the main lobe of antenna vertical pattern and within the validity range of the propagation model, i.e. from $200 \mathrm{~m}$ up to $100 \mathrm{~km}$ from the Tx. The impact of introducing this simplification depends on the antenna tilt and antenna beamwidth. Figure 4 depicts vertical antenna gain vs. distance for antenna down-tilt of $2^{\circ}$ and reveals that the impact of not considering vertical antenna pattern is smaller compared to standard deviation error between the predicted and measured signal levels, which is approximately $8 \mathrm{~dB}$, for perfectly calibrated propagation models. To solve the simplified non-linear optimization problem in a non-linear least squares sense, we neglected $F B R_{\phi}, \phi_{0}$, and $n$ in $\beta$ (4) and used the Matlab function lsqnonlin() with non-equal upper and lower optimization bounds as given in Table 1. We set the parameters of lsqnonlin() in a way that the trust-region-reflective algorithm with lower and upper bounds was selected. This was due to the fact that, first, the nature of our objective function (a sum of weighted terms including logarithmic functions) indicated that the trust-region-reflective algorithm should be effective in finding the optimum, and second, a good initial approximation together with lower and upper bounds were available from the domain knowledge of the optimization problem. Besides, the optimization procedure turned out to provide stable results on our data in a reasonable running time. In particular, the optimization bounds for parameters $A_{0}$ to $A_{3}$ were taken from [20], and $\theta_{0}$ needs to cover all possible azimuthal directions. Bounds of $G_{\max }, F B R_{\theta}$, and $m$ were extracted from antenna diagrams used by the mobile operators to cover the most typical antennas with the horizontal patterns approximately symmetrical to their bore sight direction. The starting input parameters for the function lsqnonlin() were set according to typical values in mobile networks.

Other considered parameters were selected or obtained as follows. The clutter loss information was read from the clutter database of a mobile operator, the diffraction loss was calculated from the digital elevation model as a knife-edge effect, and the Tx height was set to the average BS height of $20 \mathrm{~m}$, while the typical $\mathrm{Rx}$ height was considered to be $1.5 \mathrm{~m}$ above the ground level. Since various localization algorithms can be used to estimate the area of possible Tx locations $T$, we did not want to particularly emphasize any of them. As an example of estimating $T$ in this paper, we first performed simulations of the TA localization with a typical TA step of $553.5 \mathrm{~m}$ by using considered measurement sets. As can be seen from Figure 5, when more than ten randomly distributed measurements are available, the area of possible Tx locations $T$ on average comprises less than approximately 20 RF-REM points.

\subsection{Performance metric}

The accuracy of the constructed RF-REM reflects the performance of its construction method. This is in the literature evaluated by comparing the estimated signal levels in $\mathrm{dBm}$ of the constructed and reference RF-REM

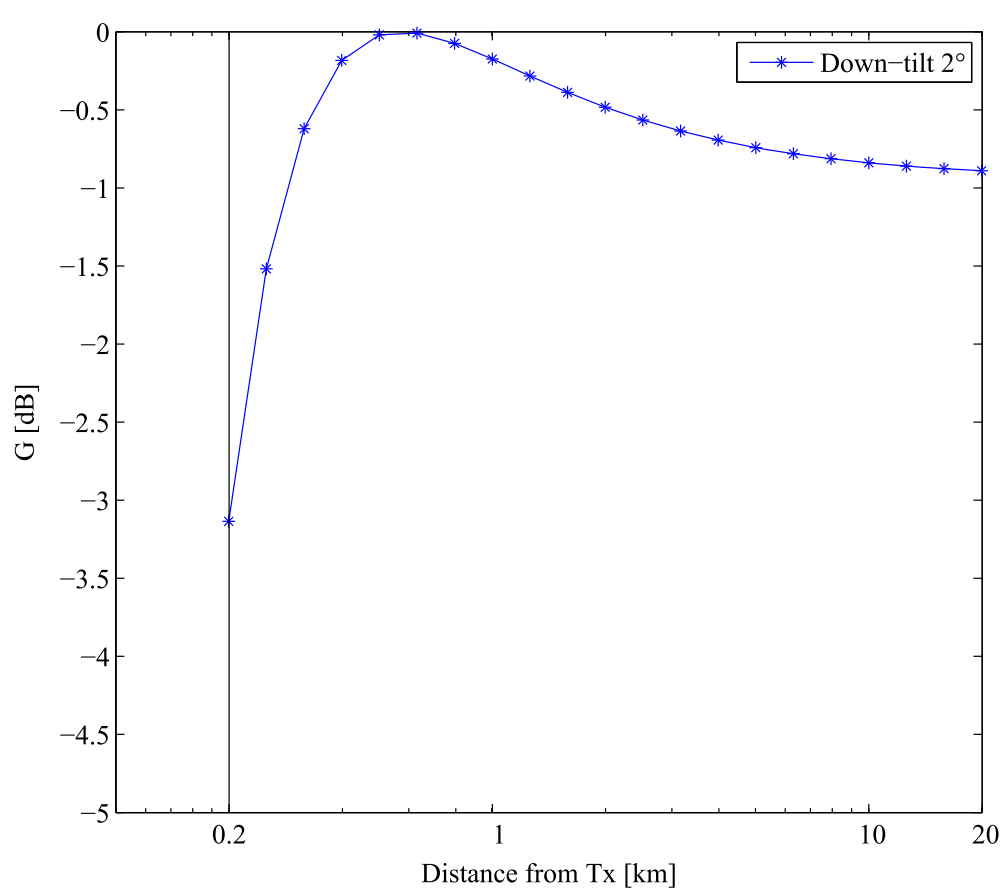

Figure 4 The impact of vertical part of antenna pattern. 
Table 1 Lower and upper optimization bounds for the STM considering the directional Tx

\begin{tabular}{|c|c|c|c|c|c|c|c|c|}
\hline & $A_{0}$ & $A_{1}$ & $A_{2}$ & $A_{3}$ & $G_{\max }[\mathrm{dBi}]$ & $F B R_{\theta}[\mathrm{dB}]$ & $\theta_{0}\left[^{\circ}\right]$ & $m$ \\
\hline Min & 20 & 40 & -20 & -10 & 2.15 & 0 & 0 & 0 \\
\hline Max & 80 & 50 & 0 & 0 & 24.4 & 77.58 & 360 & 100 \\
\hline
\end{tabular}

using the performance metrics such as mean squared error (MSE) [21], mean absolute error (MAE) [9], relative mean absolute error (RMAE) [22], root mean square error (RMSE), correct detection zone ratio $\left(\mathrm{CDZR}_{1}\right)$, and false alarm zone ratio (FAZR) $[1,8,15]$. These performance metrics largely depend on the number and distribution of measurements available for the construction, so they are not best suited for comparing RF-REM construction methods. For example, one RF-REM construction method can be more accurate than the other at a specific number of measurements, and if only the distribution of measurements is changed, the results can easily be just the opposite. Therefore, we start with comparison of RF-REM construction methods in terms of averaged RMSE for different measurement sets of the same size denoted as $\overline{\mathrm{RMSE}}$ and 95\% confidence interval (CI). Next, we estimate the $C_{D Z R}$ and FAZR curves, in order to be consistent with the works $[1,15]$.

As described in Section 4.2, 100 different distributed measurement sets for a specific size ranging from 10 to 1,000 measurements were generated. For the comparison of the methods, we first calculate RF-REM for each set and then its RMSE. Finally, the $\overline{\mathrm{RMSE}}$ and the 95\%
$\mathrm{CI}$ are calculated from RMSE values obtained from the measurement sets of the specific size, for all considered sizes. CDZR 1 and FAZR metrics are calculated only for the representative case when $N=200$ measurements are available.

\section{Performance evaluation and discussion}

In this section, we provide performance evaluation of the proposed STM and compare it to other representative RF-REM construction methods pointed out in Section 2. Simulation results on the performance of the considered methods are summarized in Figure 6 for the three selected scenarios. Results for the scenario I with randomly distributed measurements in the entire area are depicted in Figure $6 a, b$. In terms of $\overline{\mathrm{RMSE}}$ and the $95 \% \mathrm{CI}$, the results in Figure 6a show that IDW and LIvE methods have similar performance, while the IDW2 outperforms both. The performance of Kriging is comparable to the performance of IDW2, i.e. it is just slightly worse up to 1,000 measurements. STM without considering the directional antenna pattern (i.e. assuming omnidirectional transmission) in general outperforms other methods up to approximately 400 measurements, but it is outperformed by IDW2 and

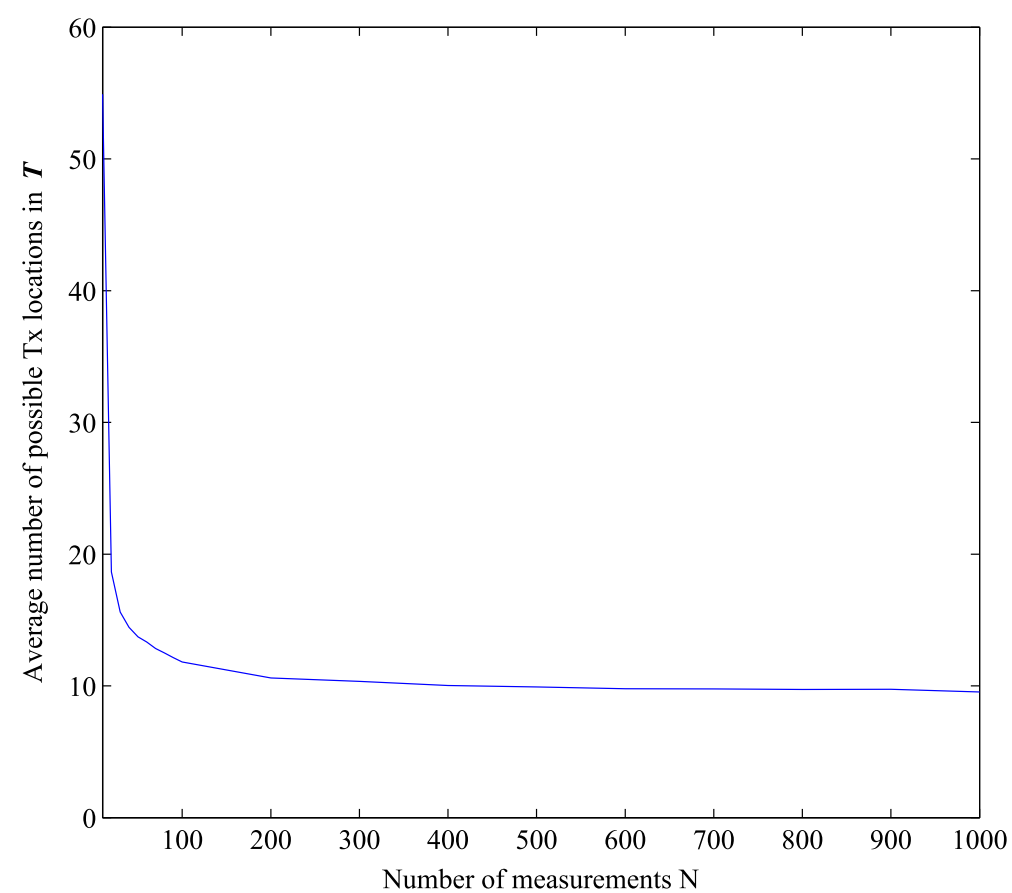

Figure 5 Average number of possible Tx locations in the area $\boldsymbol{T}$. For 100 sets with increasing number of randomly distributed measurements $N$. 

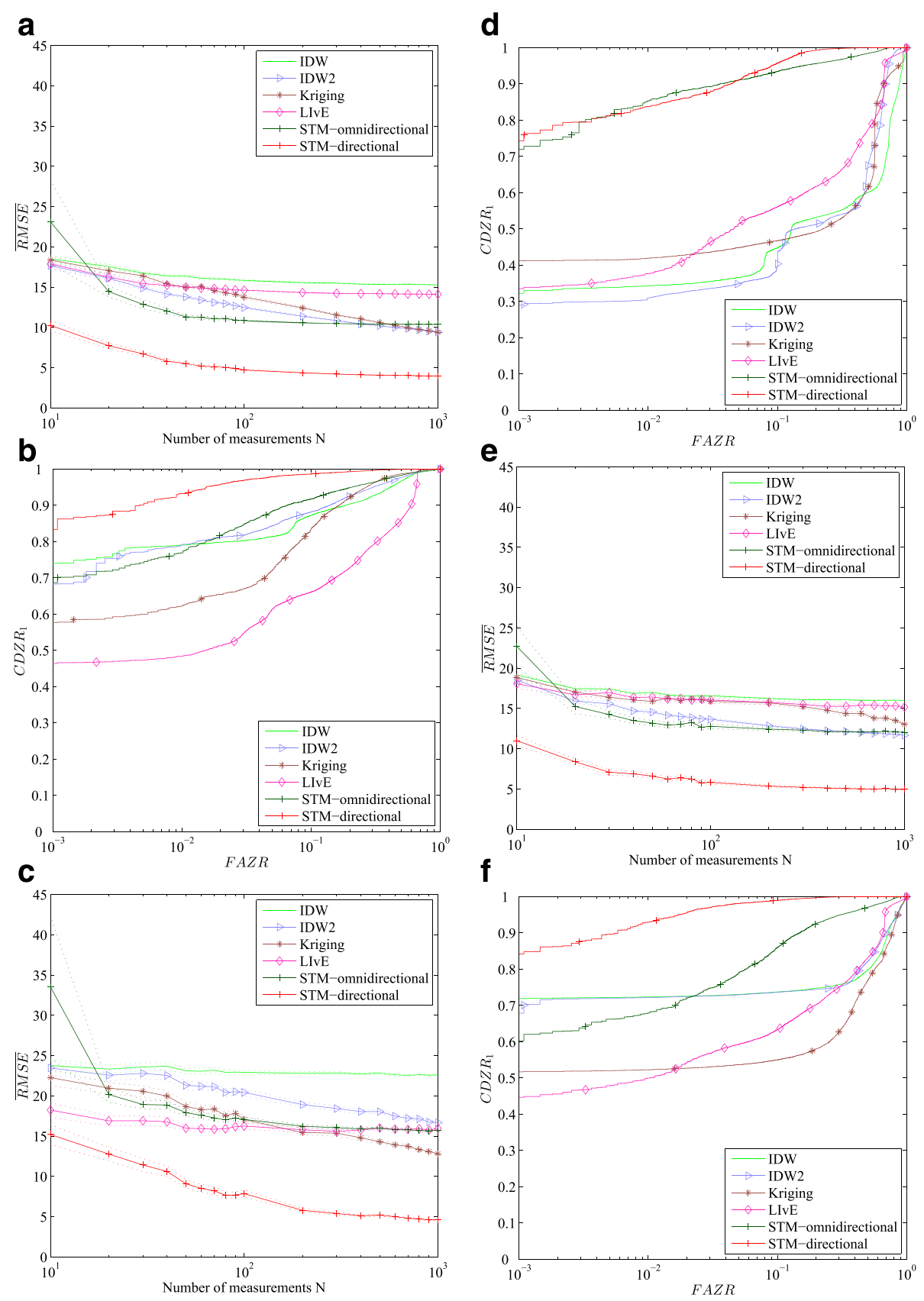

Figure 6 Results of the RF-REM construction for the sets with increasing number of randomly distributed measurements. In terms of RMSE and $95 \% \mathrm{Cl}$ for (a) scenario I, (c) scenario II, (e) scenario III. In terms of CDZR1 Vs. FAZR curves for (b) scenario I, (d) scenario II, (f) scenario III.

Kriging, when more measurement points are available. If estimating also the directional antenna pattern, STM notably outperforms all other methods for any number of measurements. The methods which do not consider antenna pattern (STM with omnidirectional antenna and LiVE) tend to reach the $\overline{\mathrm{RMSE}}$ saturation at low number of measurements $N$. Similar effect is observed for the IDW direct method, which misestimates the signal decrease rate with the distance. On the other hand, STM considering antenna pattern, IDW2 and Kriging exhibit improved performance with increasing the number of measurements.

Similar conclusions can be drawn from Figure 6b, where the performance of methods is compared in terms of receiver operating characteristics, i.e. using $C_{D Z R}$ and FAZR curves. If we consider $\mathrm{CDZR}_{1}=0.9$, meaning $90 \%$ of points in RF-REM are correctly detected as white space similar as in [1], the lowest obtained false alarm ratio 
is achieved by the STM considering directional antenna at FAZR $=0.0047$. Notably higher FAZR values, namely $0.0654,0.1394,0.1641,0.1848$, and 0.5951 , are achieved by STM considering omnidirectional antenna, IDW2, Kriging, IDW, and LIvE, respectively. Poor performance of direct methods is due to low number of measurements considered, i.e. $N=200$, while bad performance of the LIvE method can attributed to the fact that it is not considering antenna directivity.

Figure $6 \mathrm{c}, \mathrm{d}$ depicts the performance of investigated methods for the scenario II where the majority of the investigated measurements, i.e. $90 \%$, are within the main lobe of the antenna, while only some of the measurements are outside of it. In this scenario, the worst performance in terms of $\overline{\mathrm{RMSE}}$ and the $95 \% \mathrm{CI}$ is obtained for IDW, followed by IDW2. LIvE is better or similar to STM considering omnidirectional antenna and up to approximately 100 measurements better than IDW, IDW2, and Kriging. When more measurements are available, Kriging method outperforms others, except STM with directional antenna, which regardless of the number of measurements shows the best overall performance. Similarly, as in the scenario I, the direct methods which misestimate the signal decrease rate with the distance and indirect method LIvE, which does not consider the antenna directivity, exhibit $\overline{\text { RMSE }}$ saturation with the increasing number of measurements.

The results for receiver operating characteristics in scenario II in terms of $\mathrm{CDZR}_{1}$ and FAZR curves are given in Figure 6d. These results indicate that the STM notably outperform the other studied RF-REM construction methods at $\mathrm{CDZR}_{1}=0.9$, which provide significantly worse but similar FAZR, with IDW performing the worst. Comparison of the results to those obtained for the scenario I shows that all methods, except the STM with omnidirectional antenna, exhibit significant performance degradation. This is a consequence of measurements clustering in the main lobe of Tx antenna only. Additionally, the results indicate that both versions of the STM have comparable performance. This is due to less accurately estimated Tx antenna in the case of the STM with directional antenna and more accurately estimated location and parameters of the Tx in the case of the STM with omnidirectional antenna.

The results in Figure 6e,f show performance comparison of the investigated methods for the scenario III where measurements are being distributed in clusters according to clutter categories in the area of interest. In terms of $\overline{\mathrm{RMSE}}$ and the $95 \% \mathrm{CI}$, the IDW, LIvE, and Kriging methods are similar up to 200 measurements, when the performance of Kriging starts to improve and becomes comparable to the performance of IDW2 and STM with omnidirectional antenna at approximately 1,000 measurements. STM considering directional antenna demonstrates the smallest $\overline{\mathrm{RMSE}}$ regardless of the number of available measurements. The $C_{D Z R_{1}}$ vs. FAZR results in Figure $6 \mathrm{f}$ show that also in this scenario, STM considering directional antenna guarantees the best performance in finding the white space locations. At $\mathrm{CDZR}_{1}=0.9$, it achieves FAZR $=0.0058$. It is followed by STM considering omnidirectional antenna, LIvE, IDW2, IDW, and Kriging with FAZR values equal to $0.1478,0.6521,0.6932,0.7186$, and 0.7720 , respectively.

In the performance analysis, we showed that the RFREM construction is highly dependent on the spatial distribution and the number of available measurements and that ignoring the Tx antenna directivity affects all methods. Consequently, the STM with estimating the antenna directivity clearly outperforms all other methods in all considered scenarios. This reconfirms the conclusions based on our preliminary results [23] that RF-REM construction can be notably enhanced by using an indirect method, in particular with semi-empirical propagation model taking into account the characteristics of $\mathrm{Tx}$ and operating environment. The trade-off for the performance improvement is of course the increased computational complexity compared to other methods, which can be in practice efficiently addressed by parallel processing on platforms such as multi-core central processing units (CPUs), digital signal processors (DSPs), fieldprogrammable gate arrays (FPGAs), graphics processing units (GPUs), and massively parallel processor arrays (MPPAs) [24]. Generally speaking, the indirect methods are more suitable for low number of randomly distributed measurements, while the direct methods outperform the indirect ones at higher number of measurements. However, various spatial distributions and RF-REM resolutions may break this simple general rule. For example, clustering the majority of measurements in the main lobe of the Tx antenna, as assumed in the scenario II, notably decreases the performance of all methods compared to the scenario I with measurements distributed across the entire geographical area. This can be explained with the fact that the main lobe of the considered Tx antenna for obtaining reference RF-REM only covered approximately one quarter of the area of interest.

As shown on the case of the scenario III, clustering of measurements across the entire area of interest according to clutter categories yields very similar results to entirely random distribution of measurements, suggesting that realistic clustering as expected from mobile terminals could be potentially used for RF-REM construction of mobile networks BSs. Still, further research into this direction should be carried out by taking into account all BSs and coverage areas of the considered mobile operator and more realistic modelling of Rxs distribution over clutter categories. The usage of confidence intervals and in particular their width obtained for sets with low number 
of measurements indicate that the performance of the considered methods is very sensitive to the number and distribution of measurements. Moreover, evaluating the constructed RF-REM in terms of other performance metrics outlined in Section 4.4, can yield wrong interpretation of the results, as they do not take into account the different distributions of measurements which can occur in measurement sets of the specific size.

Finally, for practical implementation, it is necessary to analyse also the computational complexity of compared RF-REM construction methods. If there are $N$ measurements available in a given time moment and $M$ RF-REM locations for estimating signal levels, the computational time complexity of the IDW and IDW2 methods is $O(M N)$ [7]. If there are $K$ out of $N$ measurements within the local neighbourhood window for the ordinary Kriging interpolation, the computational time complexity of the Kriging method is $O\left(M K^{2}\right)$ [7]. The computational time complexity of the LIvE method is $O\left(M N^{3}\right)$, or $O\left(M N^{2.376}\right)$ by using Coppersmith-Winograd algorithm [8]. If the STM considering the omnidirectional antenna solves $P$ linear optimization problems, which dominate the complexity with involving the matrix inversion, its computational-time complexity is $P$ times of the LIvE method. $P$ is the number of potential Tx locations in $T$ and is dependent of the accuracy of the selected localization algorithm. On the other hand, the operation of the STM considering the Tx antenna directivity mainly depends on the selection of the non-linear optimization algorithm and its implementation. These issues were not the main focus of this paper and remain to be investigated as part of our further research, but we can note here that the price for the best performance of the STM is the highest computational-time complexity.

\section{Conclusion}

In this paper, we first presented the overall concept of REM and outlined the importance of its RF-REM layer, which is a strong tool for supporting DSA, radio resource management, and radio network planning. We focused on the RF-REM construction and proposed a new indirect method called STM. Its performance was compared to the IDW, IDW2, Kriging, and LIvE methods based on the simulated distributed measurements in a directional Tx scenario, which could in practice be obtained in a participatory manner. From the information provided by the mobile operator, we simulated the reference RF-REM of a single Tx to which the constructed RF-REMs were compared to obtain their performance in terms of $\overline{\mathrm{RMSE}}$ and $95 \% \mathrm{CI}$, as well as with $\mathrm{CDZR}_{1}$ vs. FAZR receiver operating characteristic curves, for the representative case of $N=200$ measurements. Simulation results show that the performance of RF-REM construction methods clearly varies with the number of measurements and with measurement distribution across scenarios, which can occur in practice with respect to directional Tx. It has been shown that clustering of measurements in the main lobe of the Tx antenna notably decreases the performance of all construction methods, especially of the direct methods if the application goal is finding the white spaces in the entire geographical area. Moreover, although direct RF-REM construction is more often considered than the indirect construction, it has been shown that a more accurate RF-REM can be constructed already from small number of randomly distributed measurements by using an indirect construction method with a semi-empirical propagation model taking into account the characteristics of the Tx and the operating environment. Modelling of the Tx antenna proved to be of particularly great importance, although it introduces increased computational complexity, but this can be in practice efficiently addressed by using parallel processing techniques.

\section{Abbreviations}

AA: Angle of arrival; BS: Base station; $\mathrm{CDZR}_{1}$ : Correct detection zone ratio; $\mathrm{Cl}$ : Confidence interval; CPU: Central processing unit; CR: Cognitive radio; DSA: Dynamic spectrum access; DSP: Digital signal processor; ECC: Electronic Communications Committee; FAZR: False alarm zone ratio; FCC: Federal Communications Commission; FPGA: Field-programmable gate array; GIDS: Gradient plus inverse distance squared method; GPU: Graphics processing unit; IDW: Inverse distance weighted interpolation method; IDW2: Inverse distance square weighted interpolation method; LIvE: Transmitter location estimation-based radio environment map construction method; MAE: Mean absolute error; MPPA: Massively parallel processor array; MSE: Mean squared error; MSM: Modified Shepard's interpolation method; NN: Nearest neighbour interpolation method; PSD: Power spectral density map; REELM: Radio electric exposure level map; REM: Radio environmental map; RF: Radio frequency; RFEM: Radio frequency environment map; RF-REM: Radio frequency layer of radio environment map; RMAE: Relative mean absolute error; $\overline{\mathrm{RMSE}}$ : Averaged root mean square error; RMSE: Root mean square error; Rx: Receiver; SDR: Software-defined radio; STM: Self-tuning method; TA: Timing advance; TDOA: Time difference of arrival; TOA: Time of arrival; Tx: Transmitter.

\section{Competing interests}

The authors declare that they have no competing interests.

\section{Authors' contributions}

$M P, T J$, and MM carried out most of the REM and RF-REM construction studies, developed, and tested the STM, performed simulations, interpreted the results, and drafted the manuscript. LV participated in the study of localization techniques, and AK assisted with the mathematical analysis and interpretation of the results. MŠ enabled and coordinated the access to all data of the mobile operator. All authors read and approved the final manuscript.

\section{Acknowledgements}

This work has been in part funded by the European Union from Social Fund and the FP7 projects ABSOLUTE (FP7-ICT-318632) and CREW (FP7-ICT-258301).

\section{Author details}

${ }^{1}$ Telekom Slovenije, d.d., Cigaletova 15, 1000 Ljubljana, Slovenia. ${ }^{2}$ Department of Communication Systems, Jozef Stefan Institute, Jamova cesta 39, 1000 Ljubljana, Slovenia. ${ }^{3}$ Faculty of Electrical Engineering, University of Ljubljana, Tržaška 25, 1000 Ljubljana, Slovenia. ${ }^{4}$ Jozef Stefan International Postgraduate School, Jamova cesta 39, 1000 Ljubljana, Slovenia.

Received: 27 November 2014 Accepted: 12 February 2015

Published online: 04 March 2015 


\section{References}

1. HB Yilmaz, TTugcu, Location estimation-based radio environment map construction in fading channels. Wireless Commun. Mobile Comput. 15(3), 561-570 (2013)

2. J Mitola, G Maguire, Cognitive radio: making software radios more personal. IEEE Personal Commun. 6(4), 13-18 (1999)

3. S Haykin, Cognitive Dynamic Systems Perception-action Cycle, Radar and Radio. (Cambridge University Press, Cambridge, UK, 2012)

4. ECC, Technical and operational requirements for the possible operation of cognitive radio systems in the 'white spaces' of the frequency band 470790 mhz. Ecc report 159. Electronic Communications Committee (2010) http://www.erodocdb.dk/Docs/doc98/official/pdf/ECCREP159.PDF

5. IEEE, IEEE standard for information technology - local and metropolitan area networks - specific requirements - part 22: cognitive wireless ran medium access control (MAC) and physical layer (PHY) specifications: policies and procedures for operation in the TV bands. IEEE Std 802.22-2011, 1-680 (2011). doi:10.1109/IEEESTD.2011.5951707

6. S Subramani, J Riihijarvi, B Sayrac, L Gavrilovska, M Sooriyabandara, T Farnham, P Mahonen, in Future Network Mobile Summit (FutureNetw). Towards practical REM-based radio resource management (IEEE, Warsaw, Poland, 2011), pp. 1-8

7. European FP-7 project FARAMIR reports: flexible and spectrum aware radio access through measurements and modelling in cognitive radio systems. http://www.ict-faramir.eu/index.php?id=deliverables

8. HB Yilmaz, Cooperative spectrum sensing and radio environment map construction in cognitive radio networks. PhD thesis, Bogazici University (2012)

9. D Denkovski, V Atanasovski, L Gavrilovska, J Riihijarvi, P Mahonen, in Cognitive Radio Oriented Wireless Networks and Communications (CROWNCOM), 2012 7th International ICST Conference On. Reliability of a radio environment map: case of spatial interpolation techniques (IEEE, Stockholm, Sweden, 2012), pp. 248-253

10. D Shepard, in Proceedings of the 1968 23rd ACM National Conference ACM '68. A two-dimensional interpolation function for irregularly-spaced data (ACM, New York, NY, USA, 1968), pp. 517-524

11. L Bolea, J Perez-Romero, R Agusti, in Wireless Personal Multimedia Communications (WPMC), 2011 14th International Symposium On. Received signal interpolation for context discovery in cognitive radio (IEEE, Brest, France, 2011), pp. 1-5

12. MA Azpurua, KD Ramos, A comparison of spatial interpolation methods for estimation of average electromagnetic field magnitude. Progress Electromagnetics Res. M (PIER M). 14, 135-145 (2010)

13. RJ Renka, Multivariate interpolation of large sets of scattered data. ACM Trans. Math. Softw. 14(2), 139-148 (1988)

14. EH Isaaks, RM Srivastava, An Introduction to Applied Geostatistics. (Oxford University Press, New York, 1989)

15. HB Yilmaz, T Tugcu, FFz Alagoz, S Bayhan, Radio environment map as enabler for practical cognitive radio networks. IEEE Commun. Mag. 51(12), 162-169 (2013)

16. Y Ould Isselmou, H Wackernagel, W Tabbara, J Wiart, in Antennas and Propagation, 2006. EUCAP 2006. First European Conference On. Geostatistical interpolation for mapping radio-electric exposure levels (IEEE, Nice, France, 2006), pp. 1-6. doi:10.1109/EUCAP.2006.4584752

17. BH Fleury, PE Leuthold, Radiowave propagation in mobile communications: an overview of european research. IEEE Commun. Mag. 34(2), 70-81 (1996)

18. Ericsson, TEMS cellplanner common features technical reference manual, EN/LZT 1089833 R1B (June, 2008)

19. A Hrovat, I Ozimek, A Vilhar, T Celcer, I Saje, T Javornik, Radio coverage calculations of terrestrial wireless networks using an open-source GRASS system. WTOC. 9(10), 646-657 (2010)

20. E Aarnæs, S Holm, Tuning of empirical radio propagation models effect of location accuracy. Wirel. Pers. Commun. 30(2-4), 267-281 (2004)

21. S Grimoud, B Sayrac, S Ben Jemaa, E Moulines, in Vehicular Technology Conference (VTC Fall), 2011 IEEE. Best sensor selection for an iterative rem construction (San Francisco, CA, USA, 2011), pp. 1-5. doi:10.1109/VETECF. 2011.6093004

22. M Angjelicinoski, V Atanasovski, L Gavrilovska, in Telecommunications Forum (TELFOR), 2011 19th. Comparative analysis of spatial interpolation methods for creating radio environment maps (IEEE, Belgrade, Serbia, 2011), pp. 334-337. doi:10.1109/TELFOR.2011.6143557
23. M Pesko, T Javornik, M Štular, Mohorčič, in 4th Workshop of COST Action 1C0902: Cognitive Radio and Networking for Cooperative Coexistence of Heterogeneous Wireless Networks. The comparison of methods for constructing the radio frequency layer of radio environment map using participatory measurements (Rome, Italy. 9-11 Oct 2013, 2013)

24. DB Thomas, L Howes, W Luk, in Proceedings of the ACM/SIGDA International Symposium on Field Programmable Gate Arrays. A comparison of CPUs, GPUs, FPGAs, and massively parallel processor arrays for random number generation (ACM, New York, NY, USA, 2009), pp. 63-72

\section{Submit your manuscript to a SpringerOpen ${ }^{\mathcal{O}}$ journal and benefit from:}

- Convenient online submission

Rigorous peer review

- Immediate publication on acceptance

- Open access: articles freely available online

- High visibility within the field

- Retaining the copyright to your article

Submit your next manuscript at $>$ springeropen.com 